\title{
Education and Growth with Endogenous Debt Constraints
}

\author{
David de la Croix
}

Philippe Michel

October 19, 2004

\begin{abstract}
When future human capital cannot be alienated, households are allowed to borrow up to the point where it is in their own interest not to default. In such a framework, endogenous borrowing limits arise as the outcome of individual rationality constraint. In a model where education is the engine of growth, we show that endogenous borrowing constraints imply global indeterminacy. Comparing outcomes across the various equilibria we show that the relation between growth and yields is hump-shaped. Maximum growth can arise in an equilibrium with binding borrowing constraints, specially if the elasticity of human capital to education spending is large. Deepening financial markets promotes long-run growth in the case of a poverty trap, but not necessarily otherwise.
\end{abstract}

Keywords: financial depth, borrowing constraints, indeterminacy, incentive compatibility.

JEL Classification Numbers: O410, 0160, J240, D310.

\footnotetext{
${ }^{1}$ We are grateful to Costas Azariadis, Raouf Boucekkine and Dario Maldonado for useful comments on an earlier draft.

${ }^{1}$ FNRS, Dept. of Economics and CORE, Université catholique de Louvain. David de la Croix acknowledges financial support from the Belgian French speaking community (Grant ARC 99/04-235 "Growth and incentive design") and the Belgian Federal Government (Grant PAI P5/10, "Equilibrium theory and optimization for public policy and industry regulation"). Address: David de la Croix, IRES, Place Montesquieu 3, B-1348 Louvain-la-Neuve, Belgium.

${ }^{2}$ Philippe Michel passed away on July 22, 2004. His death is a great loss for his friends and for the overlapping generations and optimal control community.
} 


\section{Introduction}

The framework proposed by Kehoe and Levine (1993) to model imperfect credit markets is becoming the benchmark to treat borrowing limits and has replaced the oldstyled set-ups where an ad-hoc liquidity constraint was imposed on the agents. In their model, default is associated to a penalty consisting in the seizure of the tangible assets of the person who has defaulted. As a consequence, this person will be excluded from financial markets for the time during which his/her assets can be seized. ${ }^{1}$

Defaulting has thus a benefit, - not reimbursing the loan -, and a cost, - being excluded from financial markets. There is in this context a borrowing limit below which it is in the interest of the households to reimburse. Lending more than this limit would inevitably lead to default. The borrowing limit depends on endogenous variables, including current and future yields. Since endogenous borrowing limits arise as the outcome of individual rationality constraints which prevent individuals from defaulting at equilibrium, enforcement of loan contracts is left to the self-interest of borrowers.

So far, the Kehoe and Levine (1993) concept was mostly used in pure exchange frameworks where savings finance consumption by other agents. For example Azariadis and Lambertini (2003) study an overlapping generations model in which endowments (which can be seen as labor income) are inalienable. Middle-aged households borrow from old-aged households to finance their consumption flow. In their set-up, changes in current and future rates of interest affect the borrowing constraints. This mechanism leads to multiple steady states, and to indeterminacy. They also show that such complex dynamics are consistent with endogenous debt limits but not with exogenous liquidity constraints.

The implications of endogenous borrowing limits for education funding have not yet been analyzed in general equilibrium, and this is the subject of this paper. We aim at modelling carefully credit market imperfections and deriving their role on education funding. This is obviously an important issue, since education is a key factor determining the ability of poor countries to grow, but it is often constrained by current resources, given the inability of students to borrow against future human capital. So far, investment in education subject to exogenous liquidity constraints has been studied by De Gregorio (1996), Buiter and Kletzer (1995), and Azariadis and de la Croix (2001). A first attempt to introduce endogenous constraints is made by Andolfatto and Gervais (2003), but they do so in an small open economy, where prices (wages and interest rates) are exogenous. They show that the usual policy scheme

\footnotetext{
${ }^{1}$ The length of exclusion from financial markets is exogenous in Kehoe and Levine (1993). Bond and Krishnamurthy (2004) go a step further by determining the level of exclusion which is required to sustain active credit markets.
} 
involving education subsidies, income taxes and pensions is welfare reducing, because pensions make borrowing constraints more binding. This is because pensions make savings for old age less useful, thereby reducing the incentive to reimburse the student loan. A richer model with four-period life agents is developed by Lochner and Monge (2002) to quantitatively assess the importance of having endogenous borrowing constraints instead of exogenous ones in the face of policy changes for the US economy. They conclude that the role of initial wealth and government subsidies is more important when borrowing constraints are endogenous.

On the empirical side, several authors have estimated the importance of borrowing constraints on education decisions. For the US, none of the methods proposed by Cameron and Taber (2004) produces evidence that borrowing constraints generate inefficiencies in the market for schooling. For developing countries, the picture is quite different. Glewwe and Jacoby (2000) show that borrowing constraints are paramount as far as private schooling expenditures are concerned (Vietnam, 1993-98). Jacoby (1994) provides some evidence of borrowing constraints in Peru. Additional references are provided by Andolfatto and Gervais (2003).

The study of education funding in a Kehoe and Levine (1993) framework gives rise to interesting questions. Having in mind a small open economy, the effect of the exogenous interest rate on education and growth is no longer straightforward, because of its complex effect on the incentive to reimburse the loan. We show that too low interest rates are bad for growth because they lead people to stay away from assets market and not reimburse their loan. Too high interest rates are bad too, because they make credit expensive. In a closed economy where total savings fund education spending, imperfect credit markets are responsible for indeterminacy of the balanced growth paths. The equilibrium where education is set at its individually optimal level may coexist with equilibria where households are credit constrained. Surprisingly, the maximum growth rate is not necessarily achieved in the situation where education is unconstrained.

In a first section we present the model. The study of the incentive constraints is provided in Section 2. The optimal education level is determined in Section 3. The above results have implications for the relationship between the interest rate and growth which are examined in Section 4. Endogenizing the interest rate, equilibrium steady states and dynamics are studied in sections 5 and 6 . A last section concludes.

\section{The model}

Each generation is composed by a continuum of agents of measure one. Population is stationary. The typical household lives for 3 periods. An agent born in $t-1$ draws 
utility from consumption when middle-aged $c_{t}$ and old $d_{t+1}$ :

$$
u\left(c_{t}, d_{t+1}\right)
$$

We assume that $u($.$) is increasing in its arguments and concave; it is homogeneous of$ degree one (homothetic preferences) and satisfies the Inada conditions.

Individuals borrow $b_{t-1}$ amount of good when young to build up their human capital for the next two periods. The workers enjoy $h_{t}$ unit of human capital when middleaged and $\delta h_{t}$ when old. The parameter $\delta$ defines the income growth ability over life, and is determined by different factors: health when old, determining the old-age endowment in efficient labor; retirement age ; effect of experience on human capital.

Human capital depends on individual investment $b_{t-1}$ and on the human capital of the previous generation $h_{t-1}$. This latter effect may reflect either the influence of parents or the society as a whole on education. For analytical tractability we assume a Cobb-Douglas function:

$$
h_{t}=A b_{t-1}^{\lambda} h_{t-1}^{1-\lambda}
$$

where $A$ is a productivity parameter and $0<\lambda<1$ is the elasticity of human capital to investment in education. The function is assumed homogeneous of degree one to be consistent with balanced growth. Defining the ratio $e_{t-1}=b_{t-1} / h_{t-1}$, human capital growth is given by:

$$
\frac{h_{t}}{h_{t-1}}=A e_{t-1}^{\lambda} \text {. }
$$

In the above framework, $b_{t-1}$ is understood as a spending on education good. One can alternatively interpret $b_{t-1}$ as a spending on physical capital: in that case, young households build their own firm by investing $b_{t-1}$ in it, and the production of this firm at time $t$ is given by $h_{t}$, and $\delta h_{t}$ at time $t+1$. The externality then reflects the influence on past production on the productivity of current capital. In the sequel we shall always interpret $b_{t-1}$ as investment in education, although an interpretation in terms of investment in physical capital is also perfectly valid.

When adult (middle-aged), an agent may choose to repay his load $b_{t-1}$ or not. If he defaults, he is kept out of the credit market for the remaining period of his life. The budget constraints in case of repayment are

$$
\begin{aligned}
c_{t} & =h_{t}-s_{t}-R_{t-1} b_{t-1} \\
d_{t+1} & =R_{t} s_{t}+\delta h_{t}
\end{aligned}
$$

$h_{t}$ and $\delta h_{t}$ represent income from labor, where the wage per unit of human capital is constant and equal to $1 . R_{t-1}$ and $R_{t}$ represents interest factors. 
In case of default, the agent is excluded from credit markets, and the budget constraints are

$$
\begin{aligned}
c_{t} & =h_{t} \\
d_{t+1} & =\delta h_{t}
\end{aligned}
$$

The problem of the consumer born in $t-1$ is to maximize its utility (1) subject to the human capital accumulation technology (2), the budget constraints (3)-(4) and the following individual rationality constraints:

1. IRC old-age: The middle-age agent are not allowed to borrow because they would never reimburse their debt when old. Hence savings should be nonnegative:

$$
s_{t} \geq 0
$$

2. IRC middle-age: The utility of repaying the debt and saving should be larger than the utility obtained from consuming labor income in each periods. This constraint can be written:

$$
\max _{s} u\left(h_{t}-s-R_{t-1} b_{t-1}, R_{t} s+\delta h_{t}\right) \geq u\left(h_{t}, \delta h_{t}\right)
$$

with $h_{t}$ given by (2).

The two conditions (5) and (6) are equivalent to the following condition bearing on $b_{t-1}$ :

$$
\max _{s \geq 0} u\left(h_{t}-s-R_{t-1} b_{t-1}, R_{t} s+\delta h_{t}\right) \geq u\left(h_{t}, \delta h_{t}\right)
$$

In this constraint, $R_{t-1}$ is observed, while $R_{t}$ is anticipated.

Firms produce a quantity $Q_{t}$ of physical goods by using efficient labor $L_{t}$ as the only input. The production function is linear: $Q_{t}=L_{t}$. The productivity of efficient labor is normalized to 1, without loss of generality given the assumptions made on technology and preferences. Perfect competition implies that marginal productivity is equal to marginal cost. The labor market equilibrium requires

$$
L_{t}=h_{t}+\delta h_{t-1}
$$

The assets market equilibrium requires

$$
b_{t-1}=s_{t-1}
$$

which determines an endogenous level for the interest rate $R_{t-1}$. 


\section{Education choices under incentive constraints}

In this section we analyze the incentive constraints in order to determine an upper bound on borrowing which will constrain - or not - the education choice. We temporarily take the interest rates $R_{t-1}$ and $R_{t}$ as given. In a first step, we analyze the constraint of non-negative savings (5) for given incomes, which amounts to study the indirect utility function. We define the value function of an unconstrained agent (indirect utility function):

$$
V\left(\omega_{1}, \omega_{2}, R\right)=\max _{s} u\left(\omega_{1}-s, \omega_{2}+R s\right)
$$

where $\omega_{1}$ is the first period income, and $\omega_{2}$ is the second period income. We also define the value function taking the constraint (5) into account:

$$
V^{+}\left(\omega_{1}, \omega_{2}, R\right)=\max _{s \geq 0} u\left(\omega_{1}-s, \omega_{2}+R s\right)
$$

The following Lemma characterizes the (unconstrained) life-cycle arbitrage conditions under the assumptions made on utility.

\section{Lemma 1}

The life-cycle arbitrage condition

$$
u_{1}^{\prime}(c, d)=R u_{2}^{\prime}(c, d)
$$

is equivalent to

$$
d / c=\mu(R),
$$

where $\mu$ is an increasing, differentiable, function from $\mathbb{R}_{++}$onto $\mathbb{R}_{++}: \mu(0)=0, \mu(+\infty)=$ $+\infty$ and $\mu^{\prime}>0$.

Proof: The life-cycle arbitrage conditions writes

$$
u_{1}^{\prime}(1, \rho)=R u_{2}^{\prime}(1, \rho)
$$

with $\rho=d / c$ (from homogeneity of degree 0 of the first-order derivatives of $u$ ). The marginal rate of substitution

$$
\operatorname{MRS}(\rho)=\frac{u_{1}^{\prime}(1, \rho)}{u_{2}^{\prime}(1, \rho)}=\frac{u_{1}^{\prime}(1 / \rho, 1)}{u_{2}^{\prime}(1, \rho)}
$$

is increasing in $\rho$ (since $u_{11}^{\prime \prime}<0$ and $u_{22}^{\prime \prime}<0$ ). The Inada conditions imply $M R S(0)=0$ and $M R S(+\infty)=+\infty$. The inverse function $\mu($.$) of M R S($.$) satisfies the properties of Lemma 1$.

We can now characterize the function $V^{+}$as follows. 


\section{Proposition 1}

If $\omega_{2} / \omega_{1}>\mu(R)$, the constraint $s \geq 0$ is binding and $V^{+}\left(\omega_{1}, \omega_{2}, R\right)=u\left(\omega_{1}, \omega_{2}\right)$. If $\omega_{2} / \omega_{1} \leq \mu(R)$, savings are given by

$$
s^{\star}=\frac{\mu(R) \omega_{1}-\omega_{2}}{R+\mu(R)} \geq 0,
$$

and the function $V^{+}\left(\omega_{1}, \omega_{2}, R\right)=V\left(\omega_{1}, \omega_{2}, R\right)$ is increasing in $R$ in the set $\{\mu(R)>$ $\left.\omega_{2} / \omega_{1}\right\}$.

Proof: Optimal savings (maximizing $V$ ) can be obtained by solving for $s$ the following identity:

$$
\frac{\omega_{2}+R s}{\omega_{1}-s}=\frac{d}{c}=\mu(R)
$$

We obtain

$$
s^{\star}=\frac{\mu(R) \omega_{1}-\omega_{2}}{R+\mu(R)} .
$$

the constraint $s \geq 0$ in the definition of $V^{+}$will not bind when $\omega_{1} / \omega_{2}>\mu(R)$. The function $V^{+}=V$ is increasing in $R$ in the set $\left\{\mu(R)>\omega_{2} / \omega_{1}\right\}$ because $s^{\star}>0$, and from the envelope theorem $\partial V / \partial R=s^{\star} u_{2}^{\prime}$.

Corollary 1 With the solution $c^{\star}=\omega_{1}-s^{\star}, d^{\star}=\omega_{2}+R s^{\star}$ maximizing $V$, we have:

$$
V\left(\omega_{1}, \omega_{2}, R\right)=\left(R \omega_{1}+\omega_{2}\right) u_{2}^{\prime}
$$

Proof: Using the homogeneity of $u$

$$
V\left(\omega_{1}, \omega_{2}, R\right)=u\left(c^{\star}, d^{\star}\right)=c^{\star} u_{1}^{\prime}+d^{\star} u_{2}^{\prime}=\left(R c^{\star}+d^{\star}\right) u_{2}^{\prime}=\left(R \omega_{1}+\omega_{2}\right) u_{2}^{\prime} .
$$

Given $\omega_{1}$ and $\omega_{2}$, Proposition 1 determines a threshold for the interest rate below which constrained savings are zero (unconstrained savings are negative or nil). The Corollary gives a useful link between $V$ and $u_{2}^{\prime}$.

We now turn on attention to the incentive constraint (IC) by making incomes $\omega_{1}$ and $\omega_{2}$ explicit: $\omega_{1}=h_{t}-R_{t-1} b_{t-1}$ and $\omega_{2}=\delta h_{t}$. Defining the debt repayment as a share of income as:

$$
x_{t}=\frac{R_{t-1} b_{t-1}}{h_{t}}=\frac{R_{t-1}}{A} e_{t-1}^{1-\lambda}
$$

we have $\omega_{1}=h_{t}\left(1-x_{t}\right)$ and the constraint (IC) can be written as:

$$
V^{+}\left(h_{t}\left(1-x_{t}\right), \delta h_{t}, R_{t}\right) \geq u\left(h_{t}, \delta h_{t}\right)
$$

which is equivalent to

$$
V^{+}\left(1-x_{t}, \delta, R_{t}\right) \geq u(1, \delta) .
$$




\section{Proposition 2}

The constraint (IC) is equivalent to an upper bound $\bar{x}_{t}$ on the income share of debt repayment $x_{t}$, given by

$$
\bar{x}_{t}=1-g\left(R_{t}\right) \text { with } g\left(R_{t}\right)=\left[\frac{u(1, \delta)}{u_{2}^{\prime}\left(1, \mu\left(R_{t}\right)\right)}-\delta\right] \frac{1}{R_{t}} .
$$

This upper bound is a function of the future interest factor $R_{t}$. It is equal to zero for low values of $R_{t}$-satisfying $R_{t} \leq R_{\text {min }}$-and it is positive for large values - satisfying $R_{t}>R_{\text {min }}-$, with $R_{\text {min }}$ such that

$$
\mu\left(R_{\min }\right)=\delta .
$$

The function $g($.$) and the threshold R_{\min }$ only depend on preferences and $\delta$.

Proof: Applying Proposition 1 to $V^{+}\left(1-x_{t}, \delta, R_{t}\right)$ we observe the following.

If $\mu\left(R_{t}\right) \leq \delta$, then $\delta /\left(1-x_{t}\right)>\mu\left(R_{t}\right)$ for all $x_{t}>0$, and we have $V^{+}=u\left(1-x_{t}, \delta\right)<u(1, \delta)$. Hence the borrowing constraint (IC) defines a maximum borrowing level of $\bar{x}_{t}=0$, implying $x_{t}=0$.

In the opposite case, when $\mu\left(R_{t}\right)>\delta$, there are positive borrowing levels $x_{t}$ such that $\delta /(1-$ $\left.x_{t}\right)<\mu\left(R_{t}\right)$, and thus $V^{+}=V$. The incentive constraint (12) will determine a borrowing limit $\bar{x}_{t}$. We rewrite equation (12) using the result (10):

$$
V=\left(R_{t}\left(1-x_{t}\right)+\delta\right) u_{2}^{\prime}\left(1, \mu\left(R_{t}\right)\right) \geq u(1, \delta) .
$$

This can be expressed as a condition on $x_{t}$ :

$$
\left(1-x_{t}\right) \geq\left[\frac{u(1, \delta)}{u_{2}^{\prime}\left(1, \mu\left(R_{t}\right)\right)}-\delta\right] \frac{1}{R_{t}} \equiv g\left(R_{t}\right) .
$$

The function $g($.$) allows to compute the borrowing limit:$

$$
x_{t} \leq 1-g\left(R_{t}\right) \equiv \bar{x}_{t} .
$$

It remains to show that the condition $x \leq 1-g=\bar{x}$ is always more restrictive than the condition $1-x>\delta / \mu$ for which $V^{+}=V$. It is straightforward to prove that at $\bar{x}$ this latter condition holds: Indeed, since $u(1, \mu)=(\mu+R) u_{2}^{\prime}(1, \mu)$, with $\mu=\mu\left(R_{t}\right)$ and $R=R_{t}$,

$$
R g+\delta=\frac{u(1, \delta)}{u(1, \mu)}(\mu+R)
$$

Since for $\delta<\mu$ we have

$$
\frac{u(1, \delta)}{u(1, \mu)}=\frac{\delta u(1 / \delta, 1)}{\mu u(1 / \mu, 1)}>\frac{\delta}{\mu}
$$

we have $R g+\delta>(\mu+R) \delta / \mu$ which leads to $g>\delta / \mu$, and thus $V^{+}=V$.

Proposition 2 provides an interesting link between the borrowing constraint and the future interest rate. Small interest rates exclude borrowing and, hence, education 
spending. Indeed, for small interest rates, households optimal consumption profile will be flatter than their income profile (condition $\delta>\mu\left(R_{t}\right)$ ), implying that their optimal level of saving is non positive. In this case, it would be optimal for them not to reimburse their education loan. As a consequence, they will not be granted access to borrowing.

According to the chosen education technology, no education spending leads to zero human capital. We could of course consider that there is a positive minimum level $\underline{h}$ of human capital reached even if households don't educate themselves, assuming for example that the human capital production function is given by:

$$
h_{t}=\max \left[\underline{h}, A e_{t-1}^{\lambda} h_{t-1}\right] \text {. }
$$

Since $g($.$) and R_{\min }$ do not depend on the education technology, Proposition 2 would still hold.

Using the link between $x_{t}$ and $e_{t-1}$ given by Equation (11), the borrowing limit $\bar{x}$ can be translated in terms of education spending $\bar{e}$ through

$$
\bar{e}_{t-1}=\left(\frac{A}{R_{t-1}} \bar{x}_{t}\right)^{\frac{1}{1-\lambda}} .
$$

Notice that $\bar{x}_{t}$ is independent from the education technology while $\bar{e}_{t-1}$ is not.

Let us study the function linking the borrowing limit to the interest rate for large interest rate, $\bar{x}(R)=1-g(R)$.

\section{Proposition 3}

The borrowing limit function $\bar{x}(R)=1-g(R)$ is increasing from 0 to 1 when $R$ goes from $R_{\text {min }}$ to $+\infty$. Its slope at $R_{\min }$ is equal to 0 . For given $R>R_{\min }, \bar{x}(R)$ decreases with respect to $\delta$.

Proof: $\bar{x}(R)$ is defined by

$$
V(1-\bar{x}, \delta, R) \equiv u\left(1-\bar{x}-s^{\star}, \delta+R s^{\star}\right)=u(1, \delta)
$$

We can show that $\bar{x}$ is increasing in $R$, i.e. $g(R)$ is decreasing in $R$ :

$$
\frac{\mathrm{d} \bar{x}}{\mathrm{~d} R}=\frac{\bar{s}^{\star} u_{2}^{\prime}}{u_{1}^{\prime}}=\frac{\bar{s}^{\star}}{R}>0,
$$

using the envelope theorem for $\partial \bar{s}^{\star}$. When $R \rightarrow R_{\min }$, we have $\bar{x}\left(R_{\min }\right)=0$ and $s^{\star}\left(R_{\min }\right)=0$. As a consequence of the latter expression,

$$
\frac{\mathrm{d} \bar{x}\left(R_{\min }\right)}{\mathrm{d} R}=0 .
$$


To compute the limit of $\bar{x}$ when $R \rightarrow+\infty$, we use the fact that the function $g$ is bounded above by the following expression:

$$
g<\frac{u(1, \delta)}{R u_{2}^{\prime}(1, \mu(R))}=\frac{u(1, \delta)}{u_{1}^{\prime}(1, \mu(R))}=\frac{u(1, \delta)}{u_{1}^{\prime}(1 / \mu(R), 1)}
$$

using the life-cycle arbitrage condition and the homogeneity of degree zero of the marginal utility. Since $\mu(+\infty)=+\infty$ and $u_{1}^{\prime}(0,1)=+\infty$, we conclude that $g$ goes to zero when $R$ goes to infinity, and

$$
\lim _{R \rightarrow \infty} \bar{x}(R)=1 .
$$

Moreover, given $R>R_{\min }, g(R)$ is increasing with respect to $\delta$, i.e.

$$
\frac{\partial g(R)}{\partial \delta}=\frac{1}{R}\left[\frac{u_{2}^{\prime}(1, \delta)}{u_{2}^{\prime}(1, \mu(R))}-1\right]>0
$$

since $\delta<\mu(R)$ and $u_{2}^{\prime}(1, \delta)>u_{2}^{\prime}(1, \mu(R))$. We also have $g(R) \geq 0$ and $g(R)<1$ since $(R+\delta) u_{2}^{\prime}(1, \mu)=V(1, \delta, R)>u(1, \delta)$ thanks to the incentive constraint, which implies

$$
R>\frac{u(1, \delta)}{u_{2}^{\prime}(1, \mu)}-\delta
$$

From this proposition we conclude that the borrowing limit decreases with the steepness of the labor income profile over time: when future labor income prospects are high, $\delta$ is high, households can borrow less.

\section{The optimal education level}

The unconstrained optimal level of education maximizes life-time income. Using the notation in de-trended terms $e_{t-1}=b_{t-1} / h_{t-1}$, the maximization problem can be written:

$$
\max _{e_{t-1}}\left(1+\frac{\delta}{R_{t}}\right) A e_{t-1}^{\lambda}-R_{t-1} e_{t-1}
$$

The optimal education is solution to the first order condition:

$$
A \lambda\left(e_{t-1}^{\star}\right)^{\lambda-1}=\frac{R_{t-1}}{1+\delta / R_{t}} .
$$

This equation determines education spending as depending negatively on both interest rates $R_{t-1}$ and $R_{t}$. 
Knowing the optimal level of education spending $e_{t-1}^{\star}$ we can compute the corresponding level of reimbursement as a share of income:

$$
x_{t}^{\star}=\frac{R_{t-1}\left(e_{t-1}^{\star}\right)^{1-\lambda}}{A}=\lambda\left(1+\frac{\delta}{R_{t}}\right) \equiv x^{\star}\left(R_{t}\right) .
$$

$x_{t}^{\star}$ is simply proportional to the factor $\left(1+\delta / R_{t}\right)$ which transforms human capital into life-cycle income.

To determine the constrained optimal level of education, it is sufficient to compare $x_{t}^{\star}$ to $\bar{x}_{t}$ to determine whether the unconstrained solution prevails or not. Both $x_{t}^{\star}=$ $x^{\star}\left(R_{t}\right)$ and $\bar{x}_{t}=\bar{x}\left(R_{t}\right)$ depend on the next period interest rate. If $x_{t}^{\star} \leq \bar{x}_{t}$, we have necessarily

$$
\frac{\delta}{1-x_{t}^{\star}} \leq \frac{\delta}{1-\bar{x}_{t}}<\mu\left(R_{t}\right)
$$

In this case savings are positive, $V^{+}=V$ and the optimum satisfies both incentive constraints. On the contrary, if $x_{t}^{\star}>\bar{x}_{t}$, the constrained optimal level is $\bar{x}_{t} \geq 0$.

Accordingly, we can write the constrained optimal level of $x_{t}$ as

$$
x_{t}=\min \left\{x^{\star}\left(R_{t}\right), \bar{x}\left(R_{t}\right)\right\} \equiv x\left(R_{t}\right) .
$$

The corresponding growth factor of the economy is:

$$
\frac{h_{t}}{h_{t-1}}=A e_{t-1}^{\lambda} \text { with } e_{t-1}=\min \left\{e_{t-1}^{\star}, \bar{e}_{t-1}\right\}
$$

\section{Proposition 4}

The constrained optimal level of borrowing is given by (17). There exists a unique level $\hat{R}>R_{\text {min }}$ equalizing the optimal income share of borrowing to the borrowing limit, i.e.

$$
\bar{x}(\hat{R})=x^{\star}(\hat{R}) \equiv \hat{x} .
$$

The borrowing constraint restricts the education choice of households if and only if $R<\hat{R}$.

Proof: From Proposition 3, the function describing the borrowing limit function $\bar{x}(R)$ is increasing from $R_{\min }$ to $+\infty$. The function $x^{\star}(R)$ given by (16) decreases from $+\infty$ to $\lambda$ when $R$ goes from 0 to $+\infty$. The decreasing function $x^{\star}(R)$ cuts only once the increasing function $\bar{x}(R)$ at some point $\hat{R}>R_{\text {min }}$.

The two functions $\bar{x}(R)$ and $x^{\star}(R)$ are plotted in Figure 1 . 


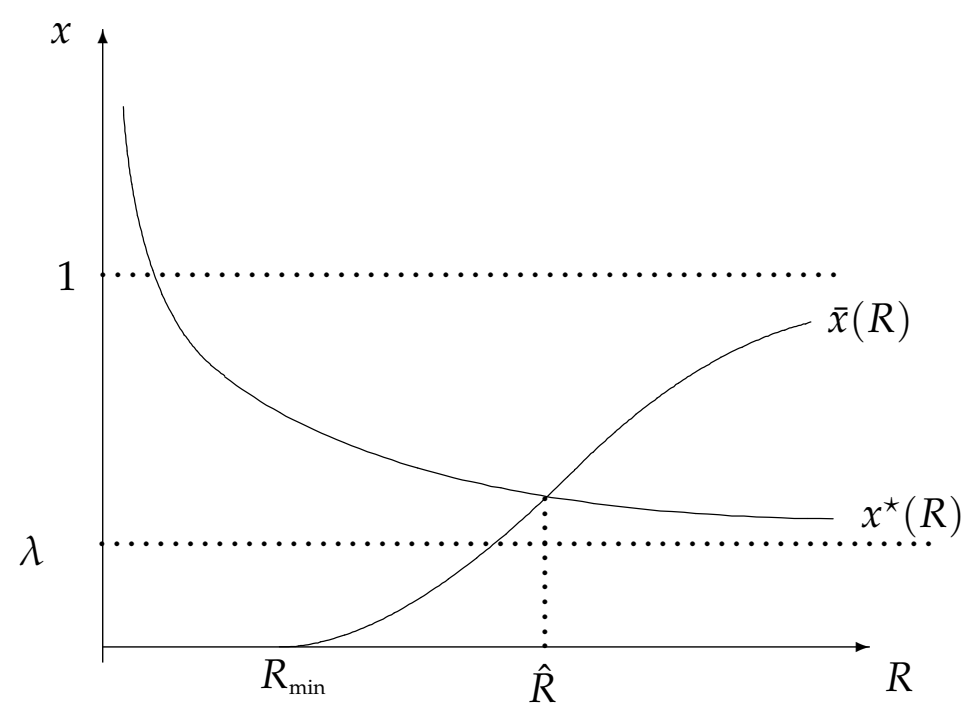

Figure 1: The functions $\bar{x}(R)$ and $x^{\star}(R)$

\section{The effect of the interest rate on growth}

Proposition 4 characterizes the share of first-period income devoted to the reimbursement of the student loan. In particular, it shows that this share is increasing in $R_{t}$ in the interval $\left[R_{\min }, \hat{R}\right]$ and does not depend on the education technology. Constrained education itself is related to both $R_{t-1}$ and $R_{t}$ through (from equation (14)):

$$
\bar{e}_{t-1}=\left(\frac{A \bar{x}\left(R_{t}\right)}{R_{t-1}}\right)^{\frac{1}{1-\lambda}} .
$$

To study the effect of interest rate on growth, we consider the case of a constant interest rate $R_{t}=R \forall t .^{2}$ This leads to:

$$
\bar{e}=\left(\frac{A \bar{x}(R)}{R}\right)^{\frac{1}{1-\lambda}} .
$$

The effect of $R$ on $\bar{e}$ is of the same sign as the effect of $R$ on $\bar{x}(R) / R$. This function admits the limit 0 both for $R \rightarrow R_{\min }$ and for $R \rightarrow+\infty$. Thus it reaches a maximum at some point $\bar{R}>R_{\min }$. $\bar{R}$ only depends on preferences and $\delta$ (since this is the case for $\bar{x})$.

\footnotetext{
${ }^{2}$ We can see this case as the one of a small open economy.
} 
Let us now consider the constrained growth rate of the economy and look at the value of the interest rate that maximizes growth. We already know that too low interest rates $\left(<R_{\min }\right)$ will go together with economic stagnation because they are incompatible with borrowing.

The maximum of the constrained growth rate cannot be reached for $R>\hat{R}$, i.e. in the interior of the unconstrained regime, because

$$
e^{\star}=\left(\frac{\lambda A(1+\delta / R)}{R}\right)^{\frac{1}{1-\lambda}}=\left(\frac{A x^{\star}(R)}{R}\right)^{\frac{1}{1-\lambda}}
$$

is decreasing in $R$. Hence, high interest rates are detrimental to growth because they depress optimal education investment. As a consequence, the growth maximizing interest rate is either equal to $\hat{R}$ (optimal investment coincides with the borrowing limit and the borrowing limit reaches a maximum at $\bar{R} \geq \hat{R}$, left panel of Figure 2), or it belongs to the interval $] R_{\min }, \hat{R}[$, and equals $\bar{R}$ (right panel of Figure 2). The general result can be stated as follows.

\section{Proposition 5}

The maximum growth rate cannot be reached in the interior of the unconstrained regime. It is attained in the interior of the constrained regime if and only if the elasticity of earnings to education, $\lambda$, is large enough. The lower bound on $\lambda$ only depends on preferences and $\delta$.

Proof: Notice first that $\bar{R}$ is independent from $\lambda$, since it corresponds to the maximum of $\bar{x}(R) / R$, itself independent from $\lambda$. The condition $\bar{R}<\hat{R}$ is equivalent to

$$
\bar{x}(\bar{R})<x^{\star}(\bar{R})=\lambda\left(1+\frac{\delta}{\bar{R}}\right) .
$$

This condition is equivalent to a lower bound on $\lambda$, which only depends on preferences and $\delta$.

The higher the value of the elasticity, the larger the optimal education, and the more likely is the maximum of the borrowing limit to be binding.

\section{Earnings Profile and Growth}

Let us now consider the effect of another important parameter which determines the slope of the earnings over life, $\delta$. As we already mentionned above, the parameter $\delta$, which defines the income growth ability over life, depends on by health, retirement age, and on the effect of age and experience on human capital. Most of the empirical literature devoted to estimate the impact of education of wages finds that age is an important factor (see Psacharopoulos (1994) for a survey). 

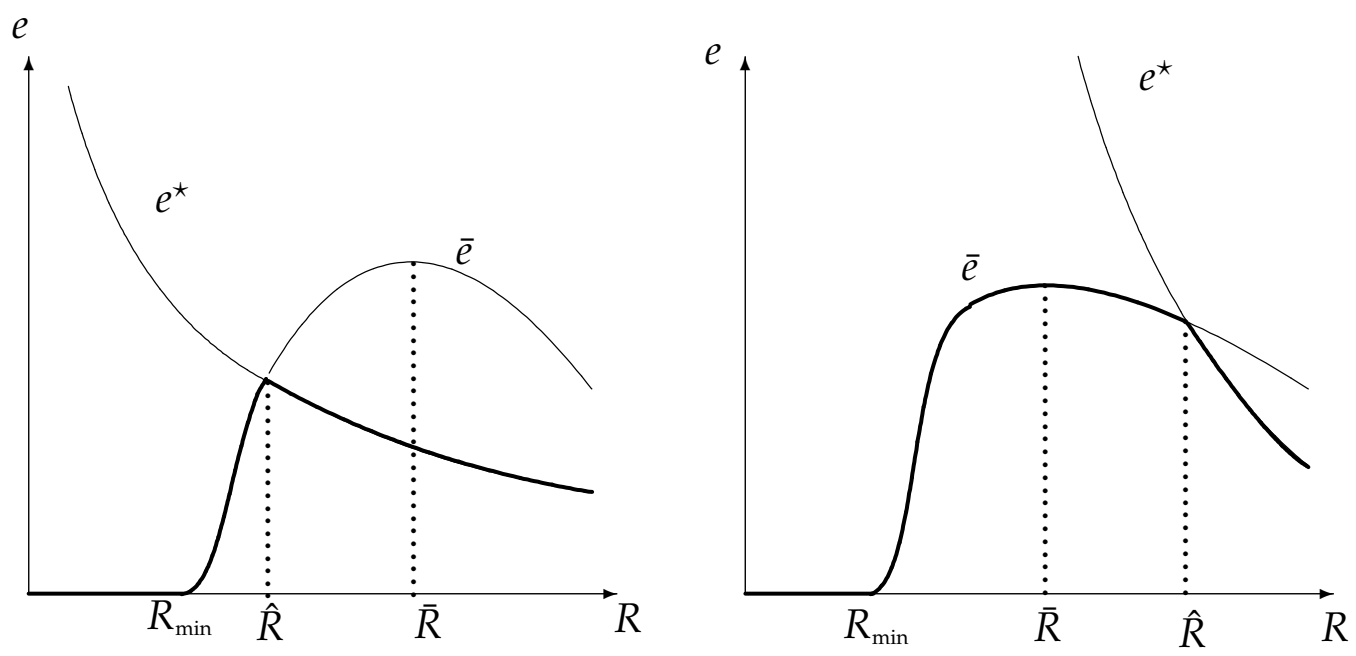

Figure 2: The maximum growth rate

If $\delta$ increases, optimal investment $x_{t}^{\star}$ increases at given $R_{t}$ (equation (16)):

$$
\frac{\partial x_{t}^{\star}}{\partial \delta}=\frac{\lambda}{R_{t}}>0
$$

According to Proposition 3, when $\delta$ is higher, households can borrow less, i.e. rationing will be more likely/severe:

$$
\frac{\partial \bar{x}_{t}}{\partial \delta}=-\frac{\partial g(R)}{\partial \delta}<0
$$

The effect of $\delta$ on growth is therefore uncertain. If the economy is credit-constrained, growth will be hampered by a rise in $\delta$, while if the constraint is not binding, growth will be enhanced by higher $\delta$.

This result may lead to interesting policy implications. For example, a policy designed to postpone the legal retirement age increases the labor endowment during the second period of life, and thereby increases $\delta$. The effect of such of policy is nonetheless uncertain, depending on the extent of borrowing constraints. Indeed, for the households who are credit-constrained, rising the retirement age would further increase the severity of the constraints, because they will be less incited to reimburse their loans if they work longer. Indeed, the penalty of being excluded from financial markets harms them less in that case. In the economy as a whole, if households are credit-constrained, long-run growth can be negatively affected by postponed retirement. This analysis can be enriched if we assume an heterogeneous population of households with different $\delta$ as in De Gregorio and Kim (2000) and Azariadis and de la Croix (2001). 


\section{The Steady State Curve}

In the previous sections we have concentrated our attention on the household decision problem, keeping the interest rate exogenous. We now consider the equilibrium condition on financial markets and look at the implied dynamics. We express the dynamics in terms of the variable $R_{t}$ starting from the definition of $\mu$ :

$$
d_{t+1}=\mu\left(R_{t}\right) c_{t}
$$

Using the budget constraints (3)-(4) and the equilibrium condition (7) we get:

$$
R_{t} b_{t}+\delta h_{t}=\mu\left(R_{t}\right)\left(\left(1-x_{t}\right) h_{t}-b_{t}\right) \text {. }
$$

Dividing by $h_{t}$ and rearranging, we obtain:

$$
\frac{b_{t}}{h_{t}}=e_{t}=\frac{\mu\left(R_{t}\right)}{R_{t}+\mu\left(R_{t}\right)}\left(1-x_{t}-\frac{\delta}{\mu\left(R_{t}\right)}\right) .
$$

Using the relationship between $e$ and $x$ given by (11) and the definition of the effective education spending (17), the dynamics of the interest rate are described by:

$$
x\left(R_{t+1}\right)=\frac{R_{t}}{A}\left(\frac{\mu\left(R_{t}\right)}{R_{t}+\mu\left(R_{t}\right)}\left[1-x\left(R_{t}\right)-\frac{\delta}{\mu\left(R_{t}\right)}\right]\right)^{1-\lambda} \equiv \frac{1}{A} \phi\left(R_{t}, x\left(x_{t}\right)\right) .
$$

This relationship holds for all $t \geq 0$. The dynamics of the economy are thus described by a first-order difference equation. $R_{t}$ is a current variable and $R_{t+1}$ is a forward looking variable. There is no pre-determined variable. ${ }^{3}$ Any path satisfying (19) is an equilibrium. There is no requirement in terms of initial condition(s). Hence, steady states are always equilibria.

Any steady state $x$ of equation (19) should satisfy:

$$
x=\frac{R^{\lambda}}{A}\left(\frac{\mu(R) R}{R+\mu(R)}\left[1-x-\frac{\delta}{\mu(R)}\right]\right)^{1-\lambda}=\frac{1}{A} \phi(R, x) .
$$

This relationship implicitly defines a function $\tilde{x}(R)$, for $R \geq R_{\min }$ (i.e. $\mu(R) \geq \delta$ ). which describes the combinations $R, x$ compatible with a steady state. Let us study this function.

\section{Lemma 2}

The steady state function $\tilde{x}(R)$ is increasing from 0 to 1 when $R$ goes from $R_{\min }$ to $+\infty$ and its derivatives satisfies: $d \tilde{x}\left(R_{\min }\right) / d R>0$.

\footnotetext{
${ }^{3}$ This simplication arises because there is no first-period consumption in the model, i.e., children do not decide separately from their parents how much to consume.
} 
Proof: The left hand side of (20) is increasing in $x$, while the right hand side is decreasing in $x$. Hence $\tilde{x}(R)$ is increasing. We also have $\tilde{x}\left(R_{\min }\right)=0$ and $\tilde{x}(+\infty)=1$.

To evaluate the slope at $R_{\min }$ we rewrite equation (20) as:

$$
x^{\frac{1}{1-\lambda}}+x N(R)=D(R)
$$

with

$$
\begin{aligned}
& N(R)=\frac{R^{\frac{\lambda}{1-\lambda}}}{A^{\frac{1}{1-\lambda}}} \frac{R \mu(R)}{R+\mu(R)} \\
& D(R)=N(R)\left(1-\frac{\delta}{\mu(R)}\right) .
\end{aligned}
$$

Differentiating the function we obtain:

$$
\left(\frac{1}{1-\lambda} x^{\frac{1}{1-\lambda}-1}+N(R)\right) \mathrm{d} x=\left(D^{\prime}(R)-x N^{\prime}(R)\right) \mathrm{d} R
$$

Evaluating this expression at $R=R_{\min }$, this simplifies to (since $x=0$ ):

$$
N\left(R_{\min }\right) \mathrm{d} x=D^{\prime}\left(R_{\min }\right) \mathrm{d} R \text { with } D^{\prime}\left(R_{\min }\right)=N\left(R_{\min }\right) \frac{\delta \mu^{\prime}\left(R_{\min }\right)}{\mu\left(R_{\min }\right)^{2}} .
$$

This finally leads to (with $\mu\left(R_{\min }\right)=\delta$ )

$$
\frac{\mathrm{d} \tilde{x}\left(R_{\min }\right)}{\mathrm{d} R}=\frac{\mu^{\prime}\left(R_{\min }\right)}{\delta}>0 .
$$

We can then analyze graphically the existence of steady states by reporting the functions $\tilde{x}(R), \bar{x}(R)$ and $x^{\star}(R)$ on the same figure, see Figure 3 . The increasing function $\tilde{x}(R)$ meets the decreasing function $x^{\star}(R)$ at a unique point $R_{u}$. This point is an equilibrium steady state if and only if $R_{u}>\hat{R} \Leftrightarrow \tilde{x}(\hat{R})<\hat{x}$. The right panel of Figure 3 represents a case with $R_{u}>\hat{R}$; the left panel represents a situation with $R_{u}<\hat{R}$. When the condition $R_{u}>\hat{R}$ holds, there also necessarily exists a steady state $R_{c}$ with $R_{\text {min }}<R_{c}<\hat{R}$ because the slope of $\tilde{x}(R)$ at $R_{\text {min }}$ is positive (Lemma 2) while the slope of $\bar{x}$ is zero (Proposition 3). One can distinguish the two cases of Figure 3 by a condition on the parameters, as it will be clear in the next section.

\section{Equilibrium dynamics}

\section{Dynamics in the poverty trap}

We first concentrate on the simple case where the equilibrium interest rate is below $R_{\text {min }}$ implying that savings and investment in education are both zero. 

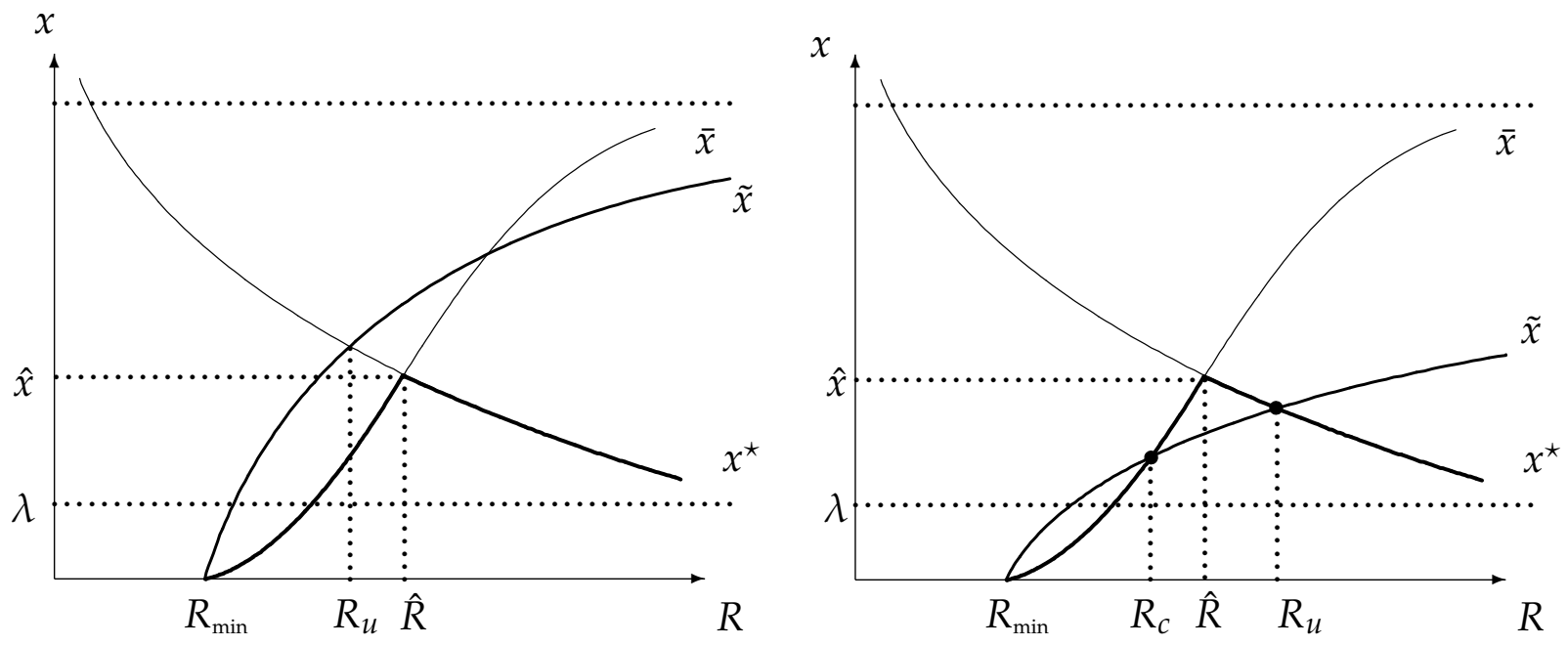

Figure 3: The steady state curve $\tilde{x}$

\section{Proposition 6}

If at date 0 all newborn households anticipate an interest factor $R_{1} \leq R_{\min }$, their constrained investment will be nil, and the equilibrium $R_{1}$ is self-fulfilled.

Proof: At date 0, old households consume their income, $d_{0}=R_{-1} s_{-1}+\delta h_{-1}$, where $s_{-1}$ and $h_{-1}$ are part of the initial conditions. Middle-aged households have a given net income $h_{0}-$ $R_{-1} s_{-1}$ where human capital $h_{0}$ and borrowing $b_{-1}$ are also part of the initial conditions. They choose $s_{0}, c_{0}$, and $d_{1}$ observing $R_{0}$. Young people chose their borrowing level $b_{0}$ observing $R_{0}$ (cost of borrowing) and anticipating $R_{1}$ (return on their future savings). If they anticipate any $R_{1} \leq R_{\min }$, they will borrow $b_{0}=0$. As a consequence, $R_{0}$ will ensure $s_{0}=b_{0}=0$. For the future, incomes are 0 , and $R_{1} \leq R_{\min }$ is an equilibrium.

\section{Dynamics in the unconstrained regime}

We now turn our attention to the case with positive savings, and study the two dynamics separely. This will allow us to characterize the local stability of the potential steady states, and to derive a condition on the parameters under which we have $R_{u}>\hat{R}$, ensuring the existence of non-trivial steady states. To keep the analysis tractable we do not consider dynamics with regime shifts.

From Equation (19), the dynamics in the unconstrained regime are given by:

$$
x^{\star}\left(R_{t+1}\right)=\frac{1}{A} \phi\left(R_{t}, x^{\star}\left(R_{t}\right)\right)
$$


Equation (21) defines a function

$$
R_{t+1}=\psi^{\star}\left(R_{t}\right) \text { with } \psi^{\star}(R)=x^{\star-1}\left(\frac{1}{A} \phi\left(R, x^{\star}(R)\right)\right) .
$$

provided that $x^{\star}\left(\right.$.) can be inversed. Since $x^{\star}$ is inversible on the interval $] \lambda,+\infty[$, the necessary and sufficient condition is

$$
\frac{1}{A} \phi\left(R_{t}, x^{\star}\left(R_{t}\right)\right)>\lambda
$$

This conditions says that if either $A$ or $\lambda$ are too large, the dynamics do not stay in the unconstrained regime and there will be a shift of regime.

Proposition 7 The function $\psi^{\star}(R)$ is defined on $[\hat{R},+\infty[$ if and only if

$$
A<\frac{1}{\lambda} \phi(\hat{R}, \hat{x}) \equiv A_{\lambda}
$$

$\psi^{\star}(R)$ is decreasing. At $\hat{R}, \psi^{\star}(\hat{R})$ is larger than, equal to, or small than $\hat{R}$, if $A$ is respectively larger than, equal to, or smaller than $\hat{A}$ with

$$
\hat{A}=\frac{\phi(\hat{R}, \hat{x})}{\hat{x}}
$$

with $\hat{x}$ given by Equation (18). A unique steady state $R_{u}>\hat{R}$ exists if and only if $A>\hat{A}$. This steady state is unstable.

Proof: The function $\phi\left(R, x^{\star}(R)\right)$ is defined if

$$
D(R) \equiv 1-x^{\star}(R)-\frac{\delta}{\mu(R)}=1-\lambda\left(1+\frac{\delta}{R}\right)-\frac{\delta}{\mu(R)}>0 .
$$

This inequality holds for $R \geq \hat{R}$. Indeed $D(\hat{R})=1-\bar{x}(\hat{R})-\delta / \mu(R)>0$, since $g(\hat{R})>\delta / \mu(\hat{R})$ by Proposition 2.

Let us study the derivative of $\phi\left(R, x^{\star}(R)\right)$.

$$
\frac{1}{\phi} \frac{\mathrm{d} \phi}{\mathrm{d} R}=\frac{1}{R}+(1-\lambda)\left(\frac{\mu^{\prime}(R)}{\mu(R)}-\frac{1+\mu^{\prime}(R)}{R+\mu(R)}+\frac{D^{\prime}(R)}{D(R)}\right) .
$$

$D^{\prime}(R) / R$ is positive and

$$
\frac{\mu^{\prime}(R)}{\mu(R)}-\frac{1+\mu^{\prime}(R)}{R+\mu(R)}>-\frac{1}{R+\mu}
$$

since $\mu^{\prime}(R)>0$ and $\mu(R)>0$. Hence

$$
\frac{1}{\phi} \frac{\mathrm{d} \phi}{\mathrm{d} R}>\frac{1}{R}-\frac{1}{R+\mu(R)}=\frac{\mu(R)}{R(R+\mu(R)} .
$$


The function $\phi\left(R, x^{\star}(R)\right)$ is thus increasing in $[\hat{R},+\infty)$.

The condition under which $\psi^{\star}$ is defined in $[\hat{R},+\infty)$ can be written:

$$
\frac{1}{A} \phi(\hat{R}, \hat{x})>\lambda \text {, i.e. } A<A_{\lambda} \text {. }
$$

Morevover, since $x^{\star}(R)$ is decreasing and $\phi\left(R, x^{\star}(R)\right)$ is increasing, $\psi^{\star}(R)$ is decreasing. At the point $\hat{R}$ we have

$$
x^{\star}\left(\psi^{\star}(\hat{R})\right)=\frac{1}{A} \phi(\hat{R}, \hat{x})=\frac{\hat{A}}{A} \hat{x}=\frac{\hat{A}}{A} x^{\star}(\hat{R}) .
$$

Hence,

$$
\psi^{\star}(\hat{R}) \gtreqless \hat{R} \Leftrightarrow x^{\star}(\psi(\hat{R})) \lesseqgtr x^{\star}(\hat{R}) \Leftrightarrow A \gtreqless \hat{A} .
$$

The steady state $R_{u}>\hat{R}$ exists if $\psi^{\star}(\hat{R})>\hat{R}$, i.e. $A>\hat{A}$.

We now study the local stability of $R_{u}$. Linearizing Equation (21), we get

$$
\frac{1}{x^{\star}\left(R_{u}\right)} \frac{\mathrm{d} x^{\star}}{\mathrm{d} R}\left(R_{u}\right) \mathrm{d} R_{t+1}=\frac{1}{\phi} \frac{\mathrm{d} \phi}{\mathrm{d} R}\left(R_{u}, x^{\star}\left(R_{u}\right)\right) \mathrm{d} R_{t} .
$$

Since we have

$$
\frac{1}{x^{\star}} \frac{\mathrm{d} x^{\star}}{\mathrm{d} R}=\frac{-\delta / R^{2}}{1+\delta / R}=-\frac{\delta}{R(R+\delta)}
$$

and

we obtain

$$
\frac{1}{\phi} \frac{\mathrm{d} \phi}{\mathrm{d} R}>\frac{\mu}{R(R+\mu)}
$$

$$
\left|\frac{\mathrm{d} R_{t+1}}{\mathrm{~d} R_{t}}\right|>\frac{\mu\left(R_{u}+\delta\right)}{\left(R_{u}+\mu\right) \delta}>1
$$

thanks to $\mu\left(R_{u}\right)>\delta$.

\section{Dynamics in the constrained regime}

We now turn our attention to the dynamics in the constrained regime. They are given by:

$$
\bar{x}\left(R_{t+1}\right)=\frac{1}{A} \phi\left(R_{t}, \bar{x}\left(R_{t}\right)\right)
$$

Equation (23) defines a function

$$
R_{t+1}=\bar{\psi}\left(R_{t}\right)
$$

if and only if

$$
\frac{1}{A} \phi\left(R_{t}, \bar{x}\left(R_{t}\right)\right)<1
$$

since $\bar{x}$ is inversible on the interval $] 0,1[$. The function $\bar{\psi}$ is given by:

$$
\bar{\psi}(R)=\bar{x}^{-1}\left(\frac{1}{A} \phi(R, \bar{x}(R))\right) .
$$


Proposition 8 The function $\bar{\psi}(R)$ is defined on $\left[R_{\min }, \hat{R}\right]$ if and only if

$$
A>\phi(\hat{R}, \hat{x}) \equiv A_{1} \text {. }
$$

$\bar{\psi}(R)$ is increasing. It satisfies $\bar{\psi}\left(R_{\min }\right)=R_{\text {min }}$ and $\bar{\psi}(R)>R$ for $R$ near $R_{\text {min }}$. At $\hat{R}, \bar{\psi}(\hat{R})$ is larger than, equal to, or small than $\hat{R}$, if $A$ is respectively smaller than, equal to, or larger than $\hat{A}$. A largest steady state $R_{c}<\hat{R}$ exists if $A>\hat{A}$. This steady state is stable.

Proof: We have that

$$
g(R)=\left[\frac{u(1, \delta)}{u(1, \mu)}(R+\mu)-\delta\right]
$$

is defined for $R \geq R_{\min }$ from Proposition 2. We deduce from

$$
\frac{\mu R}{R+\mu}\left(g(R)-\frac{\delta}{\mu}\right)=\frac{\mu u(1, \delta)}{u(1, \mu)}-\delta=\frac{u(1, \delta)}{u(1 / \mu, 1)}-\delta
$$

that

$$
\phi(R, \bar{x}(R))=R^{\lambda}\left(\frac{u(1, \delta)}{u(1 / \mu(R), 1)}-\delta\right)^{1-\lambda} .
$$

The function $\phi(R, \bar{x}(R))$ is thus increasing in $\left[R_{\min }, \hat{R}\right]$ and $\phi\left(R_{\min }, \bar{x}\left(R_{\min }\right)\right)=0$. The function $\bar{\psi}$ is thus defined and increasing in $\left[R_{\min }, \hat{R}\right]$ if and only if

$$
\frac{1}{A}<\phi(\hat{R}, \hat{x})<1 \text {, i.e. } A>\hat{A} .
$$

In a neighborhood of $R_{\min }$, for $R>R_{\min }$, we have $\tilde{x}(R)>\bar{x}(R)$ by Lemma 2 . We deduce that

$$
\bar{x}(\psi(R))=\frac{1}{A} \phi(R, \bar{x}(R))>\frac{1}{A} \phi(R, \tilde{x}(R))=\tilde{x}(R)>\bar{x}(R),
$$

since $\phi(R, x)$ is increasing with respect to $x$. Hence $\psi(R)>R$. For $A>\hat{A}$ the increasing curve $\bar{\psi}(R)$ starts from $R_{\min }$ with $\psi(R)>R$ and ends at $\psi(\hat{R})<\hat{R}$. Hence, it crosses the 45 degrees line at least once. At the largest intersection point, $R_{c}$, the slope is smaller than 1 . Hence, $R_{\min }$ is locally unstable, and $R_{c}$ is locally stable.

\section{Interpretation}

Propositions 7 and 8 define a threshold value for the productivity of education $\hat{A}$. Actual productivity can be either above or below. Figure 4 illustrates the two cases $A>\hat{A}$ and $A<\hat{A}$. In this Figure we assume that the conditions (22) and (24) are met. Otherwise, the functions are not defined for interest rates close to $\hat{R}$.

When $A<\hat{A}$, (right panel), the productivity of education is weak, and there is no non-trivial steady state. The economy does not grow. This is the standard inescapable poverty trap result, see for example de la Croix and Michel (2002). 


$$
A>\hat{A}
$$

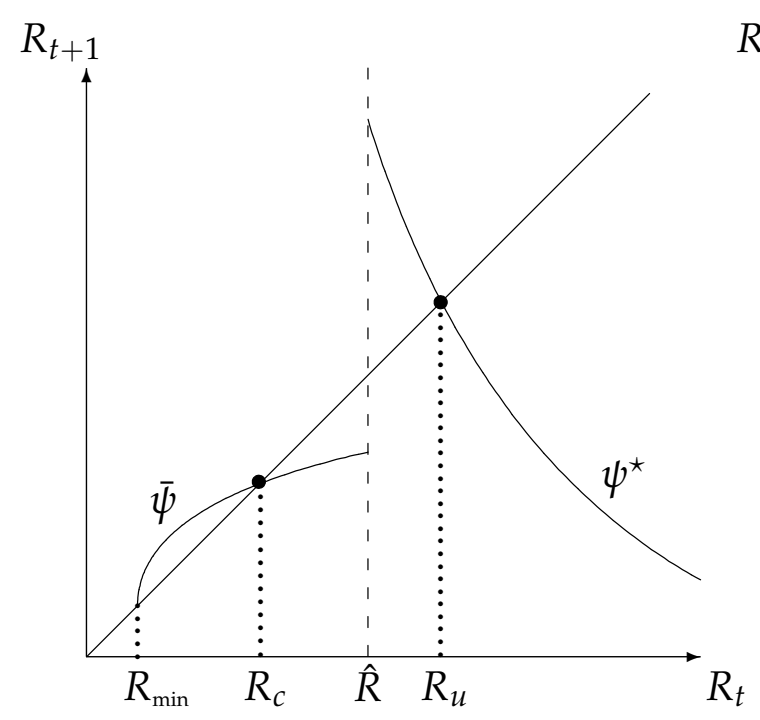

$A<\hat{A}$

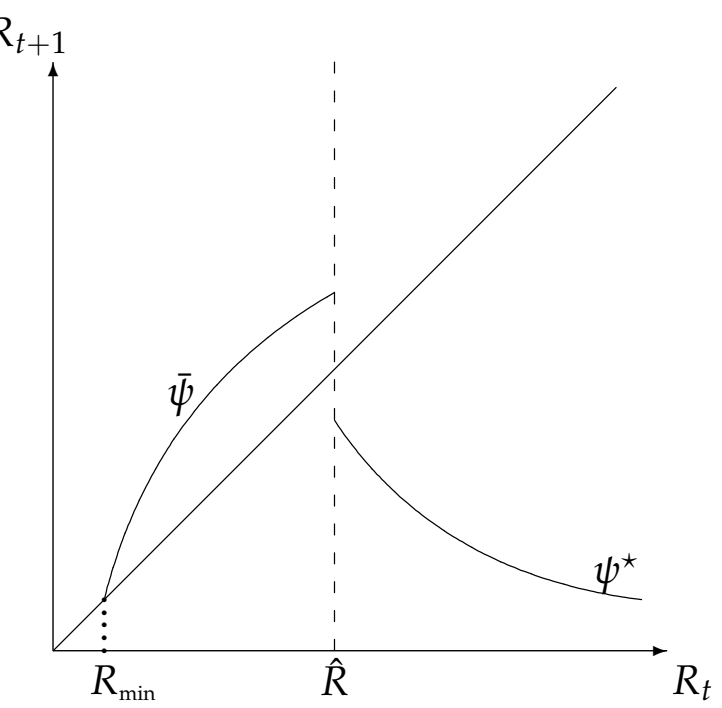

Figure 4: Two dynamics

In the case on the left, the productivity index of the learning technology $A$ is large and there are two non-trivial steady states. $R_{c}$ is in the constrained regime and it is locally stable, implying that there is an infinite number of trajectories converging to it (local indeterminacy). In addition to this steady state, there is $R_{u}$ at which investment is unconstrained. $R_{u}$ is locally unstable, hence there is a unique trajectory leading to it, the one amounting to select $R_{u}$ from the initial period onward. On the whole, there is global indeterminacy since the equilibrium can either be any $R<R_{\min }$ or any trajectory leading to $R_{c}$, or $R_{u}$ from date 0 onward.

Comparing outcomes across the various equilibria we can use the results derived in section 4 and show that the relation between growth and the interest rate is humpshaped. According to Proposition 5, maximum growth can be observed in an equilibrium where borrowing constraints are binding, specially if the elasticity of human capital to education is large.

We can now investigate what would happen if financial markets are suddenly made perfect and there is no longer borrowing constraints. Looking at Figure 3, this experiment amount to remove the curve $\bar{x}$ from the picture. Then, using Lemma 2, It is straightforward to show that there is one point for which $\tilde{x}=x^{\star}$. This steady state is by Proposition 7 unstable. This implies that when there are no borrowing limits, there is a unique equilibrium converging instantaneously to the steady state. At this 
"perfect market" steady state, growth is higher than in the poverty trap, but not necessarily than in the "imperfect market" constrained steady state. Considering the two cases of Figure 3 and the condition on $A$ that separates them (Propositions 7 and 8), it appears that moving towards perfect markets promotes growth for sure in the case on the left (poverty trap, $A$ low enough), while not necessarily in the case on the right (global inderterminacy).

\section{Conclusion}

We have introduced endogenous borrowing limits à la Kehoe and Levine (1993) in a otherwise standard OLG model with human capital. With respect to the small literature on the subject who assumes exogenous prices (Andolfatto and Gervais 2003) or solves numerically for the equilibrium (Lochner and Monge 2002), we have derived a set of useful analytical results.

If the productivity index of the learning technology is low, the economy can be catched in an inescapable poverty trap. In this case, implementing perfect credit markets makes the economy escape from stagnation.

On the contrary, if the productivity index of the learning technology is high enough, we find the same result as in Azariadis and Lambertini (2003) where multiple steady states and global indeterminacy arise as a consequence of endogenous debt limits.

Comparing outcomes across the various equilibria we show that the relation between growth and the interest rate is hump-shaped. When interest rates are low, people stay away from assets market and would not reimburse their loan. This is why banks do not lend to them, and there is no investment. With high interest rates, credit is expensive, and investment is low too. With interest rates in a medium range, households are credit constrained, but still invest positive quantities.

If the elasticity of human capital to education is high enough, maximum growth is achieved in an equilibrium where borrowing constraints are binding. In this situation, implementing a financial reform leading to perfect credit markets would reduce economic growth.

When assessing the effect of financial deepening on growth, the way borrowing constraints are modelled is key. This is the main message of the paper. The literature based on exogenous borrowing limits (see for example Aghion, Howitt, and MayerFoulkes (2004)) defends the view that there is a monotonic relationship between financial depth and long-term economic growth. This is consistent with the fact that there is a set of countries with little financial depth and slow or no growth (Africa) and another set with much better financial markets and sustained growth (OECD). Still, there is a group a countries such as China and Thailand with relatively weak 
financial markets but strong growth over the long-run. This is often view as a simple catching-up effect. Alternatively, theory says that maximum growth can be achieved in a situation where agents cannot borrow all what they want to.

\section{References}

Aghion, Philippe, Peter Howitt, and David Mayer-Foulkes. 2004. "The effect of financial development on convergence: theory and evidence." paper presented at CESifo conference, Venice.

Andolfatto, David and Martin Gervais. 2003. "Human Capital Investment and Education Policy with Endogenous Debt Constraints." paper presented at the SED 2003 conference.

Azariadis, Costas and David de la Croix. 2001. "Growth or equality? Losers and gainers from financial reform." UCLA.

Azariadis, Costas and Luisa Lambertini. 2003. "Endogenous Debt Constraints in Lifecycle Economies." Review of Economic Studies 70 (3): 461-487.

Bond, Philip and Arvind Krishnamurthy. 2004. "Regulating Exclusion from Financial Markets." Review of Economic Studies 71 (3): 681-707.

Buiter, Willem and Kenneth Kletzer. 1995. "Capital mobility, fiscal policy, and growth under self-financing of human capital formation." Canadian Journal of Economics 28 (0): s163-s194.

Cameron, Stephen and Christopher Taber. 2004. "Estimation of educational borrowing constraints using returns to schooling." Journal of Political Economy 112 (1): 132-182.

De Gregorio, Jose. 1996. "Borrowing constraints, human capital accumulation and growth." Journal of Monetary Economics 37 (1): 49-71.

De Gregorio, Jose and Se-Jik Kim. 2000. "Credit markets with differences in abilities: education, distribution and growth." International Economic Review 41 (3): 579607.

de la Croix, David and Philippe Michel. 2002. A Theory of Economic Growth: Dynamics and Policy in Overlapping Generations. Cambridge: Cambridge University Press.

Glewwe, Paul and Hanan Jacoby. 2000. "Economic Growth and the Demand for Education: Is threre a Wealth Effect ?" University of Minnesota.

Jacoby, Hanan. 1994. "Borrowing constraints and progress through school: evidence for Peru." Review of Economics and Statistics 76 (1): 151-160.

Kehoe, Timothy and David Levine. 1993. "Debt-Constrained Asset Markets." Review of Economic Studies 60 (4): 865-888. 
Lochner, Lance and Alexander Monge. 2002. "Human capital formation with endogenous credit constraints." Working Paper 8815, NBER.

Psacharopoulos, George. 1994. "Returns to Investment in Education: A Global Update." World Development 22 (9): 1325-43. 